\title{
Polyneuropathie bei Behandlung der chronischen Hepatitis B mit Vidarabin
}

\author{
K.-H. Krause, R. Raedsch, K. Brosi, K. Scheglmann und P. Berlit \\ Neurologische und Medizinische Universitätsklinik Heidelberg
}

Zwei Patientinnen mit chronischer Hepatitis B wurden 8 bzw. 12 Wochen lang mit Vidarabin in Dosen von 1000 bzw. 700 mg/d behandelt. Am Ende der Therapie litten beide distal an den Beinen unter schweren sensiblen Reizerscheinungen; klinisch war die sensible Wahrnehmung für alle Qualitäten distal an den Beinen eingeschränkt. Drei Jahre später bestanden die Störungen weiterhin, wenn auch in milderer Form. Während kurz nach Beginn der Beschwerden die sensiblen Leitgeschwindigkeiten an den unteren Extremitäten noch nicht sicher verlangsamt waren, fand sich bei beiden Patientinnen nach einem halben Jahr eine deutliche Reduktion auf Werte unter $40 \mathrm{~m} / \mathrm{s}$ für den N. suralis, die auch nach einem Jahr fortbestand. In beiden Fällen war 3 Jahre nach der Erstuntersuchung eine leichte Besserung, aber noch keine Normalisierung des elektrischen Befundes eingetreten. Der objektiv nachgewiesene lange Verlauf und die erhebliche subjektive Beeinträchtigung bei diesen Polyneuropathien sollten Anlaß zu einer sehr zurückhaltenden Indikation der Therapie mit Vidarabin sein.
Polyneuropathy in the course of treating chronic hepatitis B with adenine arabinoside (Vidarabin)

Two patients with chronic hepatitis $B$ received adenine arabinoside, at a dosage of $1000 \mathrm{mg} / \mathrm{d}$ for 8 weeks and $700 \mathrm{mg} / \mathrm{d}$ for 12 weeks, respectively. At the end of the treatment period both patients had developed severe sensory irritability in the distal parts of the legs: perception of all sensory modalities was impaired. The disorder persisted over the following three years, although in a milder form. Shortly after the onset of the symptoms sensory nerve conduction velocity in both lower legs was not definitely impaired. But six months later it was clearly reduced to below $40 \mathrm{~m} / \mathrm{sec}$ in the sural nerve, a finding which has persisted a year later. Three years after the first examination both patients are slightly improved, but conduction velocity is not yet normal. The objectively demonstrated long course and the considerable subjective complaints associated with the polyneuropathy argue for a very therapeutic use of adenine arabinoside.

\section{Kasuistik}

Fall 1: Bei der 1948 geborenen Patientin (Größe $180 \mathrm{~cm}$, Gewicht $70 \mathrm{~kg}$ ) wurden Ende 1978 erstmals erhöhte Leberwerte festgestellt, 1979 wurde bioptisch eine chronisch-aggressive Hepatitis B (HBsAntigen- und $\mathrm{HBe}$-Antigen-positiv) diagnostiziert. Nachdem ein Therapieversuch mit Cortison und Azathioprin (Imurek ${ }^{(3)}$ ) erfolglos geblieben war, erhielt die Patientin vom 20.11. bis 8. 12. 1983 insgesamt 55 Millionen Einheiten Interferon, vom 13. 12. 1983 bis 7. 2. 1984 Vidarabin-monophosphat in einer Gesamtdosis von 43,55 g. Diese Therapie konnte den entzündlichen Prozeß im Lebergewebe nicht aufhalten. Eine Laparoskopie und Leberbiopsie im November 1984 zeigten ein Fortschreiten zur Leberzirrhose mit portaler Hypertension.

Seit Ende Januar 1984 klagte die Patientin über kribbelnde und ziehende Mißempfindungen sowie ein Spannungsgefühl an den Füßen und Unterschenkeln beiderseits. Die Beschwerden nahmen bei Wärme zu, besserten sich kurzfristig beim kalten Duschen. Sie verschlimmerten sich in den folgenden Wochen, so daß am 12. 3. 1984 eine neurologische Untersuchung einschließlich einer Elektromyographie erfolgte. Klinisch fanden sich fleckförmige Bezirke mit Dysästhesie und Hypalgesie an beiden Unterschenkeln, an beiden Fußsohlen bestand eine erhebliche Hyperpathie und Dysästhesie. Die Wadenmuskulatur war druckschmerzhaft. Der Patellarsehnenreflex war rechts diskret abgeschwächt bei anamnestisch angegebenen Kreuzschmerzen mit Ausstrahlung ins rechte Bein, die Achillessehnenreflexe waren seitengleich mittellebhaft auslösbar; Lagesinn, Vibrationsempfinden und Erkennen auf die Haut geschriebener Zahlen waren normal. Elektroneurographisch fanden sich an den unteren Extremitäten grenzwertig verlangsamte Nervenleitge- 


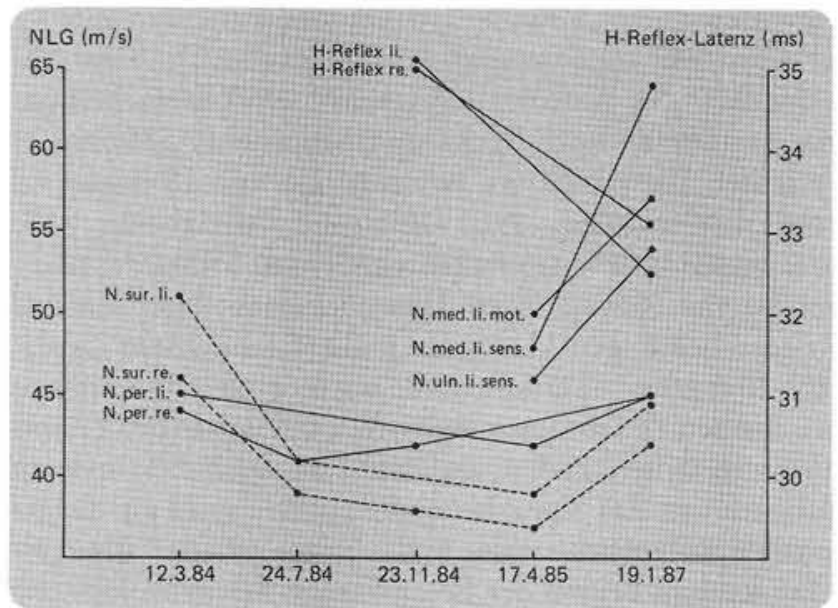

Abb. 1. Fall 1: Nervenleitgeschwindigkeiten (NLG) und Latenzen des $\mathrm{H}$-Reflexes zu den verschiedenen Untersuchungszeitpunkten.

schwindigkeiten, die bei einer Kontrolle 4 Monate später eindeutig pathologisch waren (Abbildung 1). Die neurophysiologischen Untersuchungen wurden bei dieser wie auch der zweiten Patientin mit einem DISA-1500-System durchgeführt. Die motorischen Leitgeschwindigkeiten der $\mathrm{Nn}$. peronaeus, medianus und ulnaris sowie die H-Reflex-Latenz wurden mit Hilfe von Oberflächenelektroden (Medelec EL 210 M) bestimmt, die sensiblen Leitgeschwindigkeiten des N. suralis in antidromer Technik mit Nadelelektroden (DISA $25 \mathrm{C} \mathrm{04)}$ und die der Nn. medianus und ulnaris ebenfalls in antidromer Technik mit Ringelektroden (DISA 13 L 69). Acht Monate später war der Befund unverändert. Eine elektromyographische Untersuchung des Musculus tibialis anterior rechts erbrachte einen Normalbefund, die Latenz des H-Reflexes war beiderseits verlängert. Zu diesem Zeitpunkt bestanden immer noch sehr intensive Schmerzen und Mißempfindungen an den unteren Extremitäten, die mit Liponsäure (Thioctacid ${ }^{\circledR}$ ), Neurotrat ${ }^{\circledR}$ forte sowie Cianidonal (Catergen ${ }^{\circledR}$ ) behandelt wurden; zwischenzeitlich waren auch Tramadol (Tramal ${ }^{\circledR}$ ) und Levomepromazin (Neuro$\mathrm{cil}^{\circledR}$ ) vorübergehend gegeben worden. Ohne Erfolg blieben Behandlungsversuche mit Elektrostimulation und Akupunktur.

$\mathrm{Ab}$ Anfang 1985 besserten sich die sehr starken quälenden Schmerzen hinsichtlich ihrer Frequenz; sie traten nur noch etwa einmal wöchentlich in massiver Intensität auf. Allerdings bestanden nach wie vor ständig Kribbelparästhesien, die die Patientin veranlaßten, nachts häufig auf kaltem Boden herumzulaufen. Bei einer neurologischen Untersuchung 14 Monate nach Auftreten der Beschwerden war der Achillessehnenreflex beiderseits nur noch schwach auslösbar. Es wurde eine strumpfförmige, nach distal zunehmende Hypästhesie und Hypalgesie ab Mitte der Unterschenkel angegeben, das Temperaturempfinden an beiden Vorfüßen war vermindert, das Erkennen auf die Haut geschriebener Zahlen auf beiden Fußrücken unsicher, das Vibrationsempfinden unterhalb der Knie abgeschwächt; dagegen war das Erkennen geführter Bewegungen an den Großzehen normal. Elektroneurographisch fanden sich zu diesem Zeitpunkt unverändert verlangsamte Leitgeschwindigkeiten an den unteren Extremitäten, die Latenzen des HReflexes hatten sich gegenüber der Voruntersuchung leicht gebessert (Abbildung 1). Im Musculus tibialis anterior rechts war jetzt ein leichter neurogener Umbau (mittlere Potentialdauer $11,3 \mathrm{~ms}$, mittlere Amplitude 0,9 mV, Polyphasierate $40 \%$ ) zu finden. Die motorische Leitgeschwindigkeit des Nervus medianus links war grenzwertig, ebenso die distalen sensiblen Latenzen sowie Leitgeschwindigkeiten der Nervi medianus und ulnaris links.

Bei der letzten Nachuntersuchung im Januar 1987 klagte die Patientin weiterhin über intermittierende, mehrmals täglich einige Sekunden lang anhaltende kribbelnde Mißempfindungen in den Unterschenkeln und Füßen; zeitweise habe sie das Gefühl, als ob die Haut platze; die Beschwerden nähmen bei Wärme zu. Öfters wache sie nachts auch wegen der Mißempfindungen in den Beinen auf. Eine Veränderung der Schweißsekretion an den Beinen habe sie nicht bemerkt, dagegen sei ihr aufgefallen, daß an zwei Arealen an beiden Unterschenkeln lateral vom Schienbein kein Haarwuchs mehr bestehe. Der Achillessehnenreflex war beiderseits unverändert nur schwach auslösbar, der Befund bei der sensiblen Prüfung war ebenfalls gleichgeblieben. Neurographisch fand sich eine Besserung der Leitgeschwindigkeiten, insbesondere des Nervus suralis beiderseits, die aber immer noch grenzwertig erniedrigt waren, sowie der Leitgeschwindigkeiten an den oberen Extremitäten im Vergleich zur Voruntersuchung, auch die Latenz des H-Reflexes war gebessert (Abbildung 1).

Fall 2: Die 1950 geborene Patientin (Größe $170 \mathrm{~cm}$, Gewicht 54 $\mathrm{kg}$ ) wurde im April 1981 erstmals wegen einer Hepatitis B (HBsund $\mathrm{HBe}$-Antigen-positiv) behandelt, zunächst ein Jahr mit Azathioprin und 21/4 Jahre mit Cortison. Ab November 1983 erhielt sie für 3 Wochen Interferon (Gesamtdosis 17 Millionen Einheiten), anschließend vom 13.12. 1983-20. 2. 1984 Vidarabin-phosphat in einer Gesamtdosis von 41,2 g. Bei der Patientin kam es im weiteren Verlauf zur Serokonversion (HBe-Antigen zu Anti-HBe) und Besserung aller entzündlichen Leberparameter, 1985 war der histologische Leberbefund vollständig normalisiert.

Etwa eine Woche nach Beendigung der Therapie mit Vidarabin spürte die Patientin beiderseits brennende und stechende, tags und nachts gleich intensive Schmerzen, von den Knien bis in alle Zehen ausstrahlend, außerdem eine Pelzigkeit an der Wadeninnenseite beiderseits und an den Füßen. Bei einer neurologischen Untersuchung im März 1984 fanden sich entsprechende sensible Störungen, das Vibrationsempfinden an den Vorfüßen war herabgesetzt. Der Achillessehnenreflex war seitengleich gut auslösbar. Elektroneurographisch war zu diesem Zeitpunkt kein sicher pathologischer Befund zu erheben (Abbildung 2). Drei Monate später fanden sich dagegen eine deutliche Abnahme der sensiblen Leitgeschwindigkeiten an den unteren Extremitäten, eine Zunahme der Latenz des H-Reflexes sowie leicht erniedrigte motorische Leitgeschwindigkeiten (Abbildung 2). Therapeutisch bekam die Patientin anfangs Tramadol (Tramal ${ }^{\sqrt{8}}$ ) und Levomepromazin (Neurocil ${ }^{8}$ ), dann Liponsäure (Thioctacid ${ }^{8}$ ) und Pyridoxin (Benadon ${ }^{8}$ ). Versuche mit Akupunktur schlugen fehl. Die Schmerzen in den Beinen ließen ein halbes Jahr nach Beginn etwas nach, waren aber in wechselnder Intensität nahezu ständig vorhanden. Am schlimmsten seien die Beschwerden nachts bei Bettwärme. Eine weitere Abnahme der Leitgeschwindigkeit des Nervus suralis links zeigte sich neurographisch im November 1984, die Latenz des H-Reflexes war rechts grenzwertig, links leicht verlängert (Abbildung 2). Das

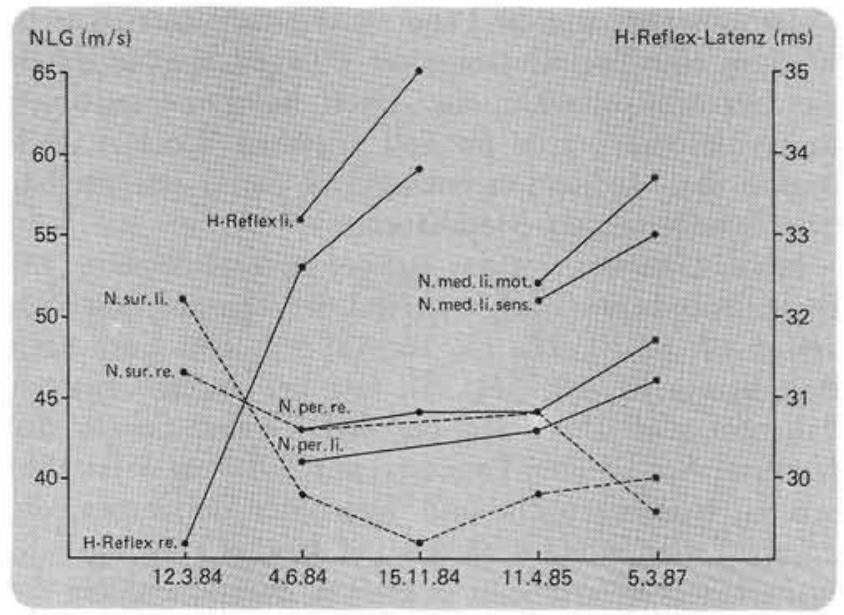

Abb. 2. Fall 2: Nervenleitgeschwindigkeiten (NLG) und Latenzen des $\mathrm{H}$-Reflexes zu den verschiedenen Untersuchungszeitpunkten. 
Elektromyogramm des Musculus tibialis anterior rechts war zu diesem Zeitpunkt unauffällig. Bei einer neurologischen Kontrolluntersuchung im April 1985 war der Achillessehnenreflex beiderseits nur noch schwach auslösbar, sensibel bestanden strumpfförmige Hypalgesie und Hypästhesie ab Mitte beider Unterschenkel sowie Therm- und Pallhypästhesie an den Vorfüßen, während der Lagesinn an den Großzehen gut erhalten war. Neurographisch zeigte sich keine wesentliche Änderung im Vergleich zum Vorbefund (Abbildung 2), elektromyographisch war bei Fehlen der pathologischen Spontanaktivität ein leichter neurogener Umbau (mittlere Potentialdauer $12,2 \mathrm{~ms}$, mittlere Amplitude $1,0 \mathrm{mV}$, Polyphasierate $50 \%$ ) im Musculus tibialis anterior links sowie im Musculus extensor hallucis longus rechts nachzuweisen.

Drei Jahre nach Beginn der Beschwerden gab die Patientin für die Zwischenzeit nur eine unwesentliche Besserung an, sie sei immer noch stark durch die Mißempfindungen beeinträchtigt. Der klinische Befund war im Vergleich zur Voruntersuchung unverändert. Neurographisch waren die sensiblen Leitgeschwindigkeiten an den unteren Extremitäten verlangsamt (Abbildung 2). Elektromyographisch fiel neben dem bekannten Befund im Musculus tibialis anterior links im Musculus extensor digitorum brevis links pathologische Spontanaktivität in Form von Fibrillieren und positiven scharfen Wellen auf, bei maximaler Willkürinnervation rekrutierte ein Interferenz- bis Übergangsmuster mit meist neurogen umgebauten, bis $8 \mathrm{mV}$ hohen Muskelaktionspotentialen.

\section{Diskussion}

Bei beiden Patientinnen war der zeitliche Zusammenhang zwischen der Vidarabin-Therapie und dem Auftreten polyneuropathischer Beschwerden evident. Die Tatsache, daß unsere Patientinnen vor der Behandlung mit Vidarabin über keinerlei polyneuropathische Symptome geklagt hatten, spricht gegen eine Verursachung der Polyneuropathie durch die Hepatitis B. Hierbei sind Polyneuropathien zum einen extrem selten, zum anderen äußern sie sich überwiegend in einer schweren Demyelinisierung (19), wie sie bei unseren Patientinnen nicht vorlag. Inwieweit die Grundkrankheit zur Ausprägung und Persistenz der Polyneuropathie beigetragen haben könnte, muß natürlich offenbleiben. Hinsichtlich der zentralnervösen Nebenwirkungen unter Vidarabin wurde festgestellt, daß eine Leberschädigung für deren Manifestation keinen entscheidenden Faktor darstellt (3). Bei unserer zweiten Patientin, bei der nach der Vidarabin-Therapie die Leberwerte normalisiert waren, scheidet eine Beeinflussung der Polyneuropathie durch die Hepatitis ohnehin aus. Über die ersten sensiblen Reizsymptome wurde im Fall 1 sieben Wochen nach Beginn der Medikation geklagt, im Fall 2 unmittelbar nach Beendigung der Medikation.

In der Literatur trat das "severe pain syndrome « (15) ebenfalls teilweise noch während der Therapie mit Vidarabin $(4,6,10,12,13,16,18)$ auf, teils kurz nach Beendigung $(5,10,16,18)$, wie bei unserer zweiten Patientin. Die Dosis lag bei den meisten der in der Literatur mitgeteilten Patienten mit 5-10 mg Vidarabin pro kg Körpergewicht und Tag unter derjenigen, die unsere Patientinnen erhielten (11 bzw. $13 \mathrm{mg} / \mathrm{kg} \cdot \mathrm{d}$ ). Bemerkenswert erscheint in diesem Zusammenhang die Beobachtung von Smith und Mitarbeitern (18), die beim Vergleich einer Vidarabin-Dosis von $5 \mathrm{mg} / \mathrm{kg} \cdot \mathrm{d}$ bei sechs
Patienten und $7,5 \mathrm{mg} / \mathrm{kg} \cdot \mathrm{d}$ bei vier Patienten nur im letzteren Fall ein Schmerzsyndrom fanden. Die Dosisabhängigkeit des Auftretens der sensiblen Reizerscheinungen bestätigten auch Sacks und Mitarbeiter (15) an 29 Patienten, die mit Dosen zwischen 2,5 und $15 \mathrm{mg} / \mathrm{kg} \cdot \mathrm{d}$ behandelt wurden. Preiksaitis und Mitarbeiter (13) berichteten über einen Patienten, der am frühesten, nämlich bereits 10 Tage nach Therapiebeginn, über sensible Reizerscheinungen klagte; er hatte die höchsten Dosen $(15-20 \mathrm{mg} / \mathrm{kg} \cdot \mathrm{d})$ erhalten, die auch deutlich über denen bei unseren Patientinnen lagen. Wie bei den in der Literatur mitgeteilten Fällen, äußerte sich auch bei unseren Patientinnen das Krankheitsbild überwiegend als distale sensible Polyneuropathie. Festzuhalten ist, daß übereinstimmend zu Beginn der Beschwerden der neurologische Befund noch als weitgehend unauffällig geschildert wird. In einigen Fällen änderte sich dies bis zum Verschwinden der Beschwerden nach einigen Wochen nicht, in anderen wurden, wie bei unseren Patientinnen, im weiteren Verlauf eindeutige Symptome einer distalen Polyneuropathie nachweisbar. Daß hierbei nicht ausschließlich sensible Fasern betroffen sind, belegen die elektromyographischen Befunde mit Ausbildung einer chronischen neurogenen Schädigung im Musculus tibialis anterior bei beiden Patientinnen sowie florider Denervierung im kurzen Zehenstrecker bei einer von beiden. Hinweise auf eine neurogene Schädigung der Beinmuskulatur im Elektromyogramm fanden sich gleichfalls bei dem Patienten von Chauplannaz und Mitarbeitern (4) sowie Feldman und Mitarbeitern (5). Hoofnagle und Mitarbeiter (6) sowie Sacks und Mitarbeiter (16) beschrieben dagegen ein unauffälliges Elektromyogramm, während Lok und Mitarbeiter (10) nur Angaben zur Neurographie machten, die eine überwiegend sensible Polyneuropathie bestätigte. Eine Besonderheit stellt die von uns bei einer Patientin eindeutig nachgewiesene Mitbeteiligung der oberen Extremitäten dar. Interessant erscheint auch der von einer unserer Patientinnen angegebene Haarausfall an den unteren Extremitäten, der als Hinweis auf eine Schädigung vegetativer Fasern bei chronischer Polyneuropathie zu deuten sein dürfte; entsprechende Befunde konnten wir kürzlich bei Patienten mit längerdauernder Meralgia paraesthetica im betroffenen Hautareal erheben (8).

Im Vergleich mit den bisher in der Literatur mitgeteilten Fällen war der Verlauf bei unseren Patientinnen außerordentlich langwierig; noch 3 Jahre nach Beginn der Symptome waren beide nicht beschwerdefrei. Während sich im einen Fall die Leitgeschwindigkeiten nach dieser Zeit wieder weitgehend normalisiert hatten, waren sie im anderen Fall immer noch eindeutig pathologisch. In der Literatur wurden entsprechende neurographische Verlaufskontrollen unseres Wissens bisher nicht mitgeteilt. Hinsichtlich der Dauer der Beschwerden geben manche Autoren einige Wochen bis zu 9 Monaten an (5, $6,12,13,15)$. Von den sieben Patienten von Lok und Mitarbeitern (10) waren fünf nach 7-30 Wochen 
beschwerdefrei, bei zwei Patienten persistierten die Beschwerden über diese Zeit hinaus. Smith und Mitarbeiter (18) beschrieben zum Teil länger als 3 Monate dauernde Beschwerden; Chauplannaz und Mitarbeiter (4) beobachteten ihren Patienten ein Jahr lang, ohne daß es bis dahin zu einer Restitution gekommen war. Es scheint also so, daß sich bei einigen Patienten eine sehr hartnäckige chronische Polyneuropathie nach VidarabinGabe entwickeln kann. Generell war die subjektive Beeinträchtigung bei unseren Patientinnen sehr ausgeprägt und lange anhaltend. Daher sollte die Indikation zu einer Therapie mit Vidarabin nur äußerst zurückhaltend gestellt werden. Auf die Bedeutung der Dosierung wurde bereits hingewiesen: Tagesdosen von $5 \mathrm{mg} / \mathrm{kg}$ Körpergewicht sollten nach Möglichkeit nicht überschritten werden. Generell bleibt abzuwarten, ob sich Vidarabin überhaupt als Mittel zur Therapie der chronischen Hepatitis B behaupten kann (2).

\section{Literatur}

1 Bassendine, M. F., R. G. Chadwick, J. Salmeron, U. Shipton, H. C. Thomas, S. Sherlock: Adenine arabinoside therapy in HBsAg-positive chronic liver disease. A controlled study. Gastroenterology 80 (1981), 1016.

2 Becker, H. J.: Vidarabin (Adenin-Arabinosid) in der Therapie der chronischen HBsAg-positiven Hepatitis. Biochemische Grundlagen und gegenwärtiger Stand der Therapie. Inn. Med. 13 (1986), 237.

3 Burdge, D. R., A. W. Chow, S. L. Sacks: Neurotoxic effects during vidarabine therapy for herpes zoster. Canad. med. Ass. J. 132 (1985), 392.

4 Chauplannaz, G., C. Trepo, A. M. Brunon, B. Bady: Neuropathie après traitement d'une hépatite chronique active par vidarabine. Rev. Neurol. (Paris) 140 (1984), 743

5 Feldman, S., P. K. Robertson, L. Lott, D. Thornton: Neurotoxicity due to adenine arabinoside therapy during varicella-zoster virus infections in immunocompromised children. J. infect. Dis. 154 (1986), 889.

6 Hoofnagle, J. H., R. G. Hanson, G. Y. Minuk, C. S. Pappas, D. F. Schafer, G. M. Dusheiko, S. E. Straus, H. Popper, E. A. Jones: Randomized controlled trial of adenine-arabinoside monophosphate for chronic type B hepatitis. Gastroenterology 86 (1984), 150.

7 Hoofnagle, J. H., G. Y. Minuk, G. M. Dusheiko, D. F. Schafer, R. Johnson, S. Straus, E. A. Jones, J. L. Gerin, K. Ishak: Adenine arabinoside 5'-monophosphate treatment of chronic type B hepatitis. Hepatology 2 (1982), 784 .

8 Krause, K.-H., M. Flemming, K. Scheglmann: Alopezie bei der Meralgia paraesthetica. Hautarzt 38 (1987), 474.

9 Lauter, C. B., E. J. Bailey, A. M. Lerner: Microbiologic assays and neurological toxicity during use of adenine arabinoside in humans. J. infect. Dis. 134 (1976), 75
10 Lok, A. S. F., L. A. Wilson, H. C. Thomas: Neurotoxicity associated with adenine arabinoside monophosphate in the treatment of chronic hepatitis B virus infection. J. antimicrob. Chemother. 14 (1984), 93.

11 Nadel, A. M.: Vidarabine treatment for herpes simplex encephalitis. Development of unusual tremor during treatment. Arch. Neurol. (Chic.) 38 (1981), 384.

12 Perillo, R. P., F. G. Regenstein, C. J. Bodicky, C. R. Campbell, G. E. Sanders, Y. C. Sunwoo: Comparative efficacy of adenine arabinoside 5' monophosphate and prednisone withdrawal followed by adenine arabinoside 5 '-monophosphate in the treatment of chronic active hepatitis type B. Gastroenterology 88 (1985), 780.

13 Preiksaitis, Y. K., B. Lang, P. K. Ng, L. Brox, G. A. Lepage, D. L. Tyrell: Effect of liver disease on pharmacokinetics and toxicity of 9- $\beta D$-arabinofuranosyladenine-5' $\beta$-monophosphate. J. infect. Dis. 114 (1981), 358.

14 Ross, A., A. Julia, C. Balakrishman: Toxicity of adenine arabinoside in humans. J. infect. Dis. 133, Suppl. (1976), 192.

15 Sacks, S. L., G. H. Scullard, R. B. Pollard, P. B. Gregory, W. S. Robinson, T. C. Merigan: Antiviral treatment of chronic hepatitis B virus infection. Pharmacokinetics and side effects of interferon and adenine arabinoside alone and in combination. Antimicrob. Agents Chemother. $21(1982), 93$.

16 Sacks, S. L., J. L. Smith, R. B. Pollard: Toxicity of vidarabine. J. Amer. med. Ass. 241 (1979), 28.

17 Scullard, G. H., R. B. Pollard, J. L. Smith, S. L. Sacks, P. B. Gregory, W. S. Robinson, T. C. Merigan: Antiviral treatment of chronic hepatitis B virus infection. Changes in viral markers with interferon combined with adenine arabinoside. J. infect. Dis. 43 (1981), 772.

18 Smith, C. I., L. W. Kitchen, G. H. Scullard, W. S, Robinson, P. B. Gregory, T. C. Merigan: Vidarabine monophosphate and human leukocyte interferon in chronic hepatitis B infection. J. Amer. med. Ass. 247 (1982), 2261.

19 Tsukada, N., C.-S. Koh, A. Inoue, N. Yanagisawa: Demyelinating neuropathy associated with hepatitis $B$ virus infection. Detection of immune complexes composed of hepatitis B virus surface antigen. J. neurol. Sci. 77 (1987), 203.

20 Van Etta, L., J. Brown, A. Mastin, T. Wilson: Fatal vidarabine toxicity in a patient with normal renal function. J. Amer. med. Ass. 246 (1981), 1703.

21 Weller, I. V. D., M. F. Bassendine, A. Craxi, M. J. F. Fowler, J. Monjardino, H. C. Thomas, S. Sherlock: Successful treatment of HBs and $\mathrm{HBe}-\mathrm{Ag}$ positive chronic liver disease. Prolonged inhibition of viral replication by highly soluble adenine arabinoside 5'-monophosphate (ARA-AMP). Gut 23 (1982), 717.

Privatdozent Dr. K.-H. Krause, Dr. K. Brosi, K. Scheglmann, Privatdozent Dr. P. Berlit Neurologische Universitätsklinik Im Neuenheimer Feld 400 6900 Heidelberg

Privatdozent Dr. R. Raedsch Medizinische Universitätsklinik, Abteilung IV Bergheimer Str. 58 6900 Heidelberg 